\title{
Management of patients with sexually transmitted infections by general practitioners in the district of Gampaha
}

\author{
Jayakody W.C.J.K. ${ }^{1}$, Pathmeswaran A. ${ }^{2}$, Wickramasuriya C.D. ${ }^{3}$
}

\begin{abstract}
Introduction: In Sri Lanka, care for sexually transmitted infections (STIS) is given by both public and private sectors. Though there is an organized system for provision of care in government STD clinics, system available for the same purpose in private sector has not been studied.
\end{abstract}

Objective: To describe the knowledge and practices related to the management of patients presenting with symptoms and signs of sexually transmitted infections (STD Syndromes) among general practitioners in the District of Gampaha.

Method: A descriptive cross sectional study was carried out among 200 general practitioners. Data were collected by using a self-administered structured questionnaire in English language and analyzed using SPSS 16.

Results: Majority of the study population were male (76\%) with MBBS qualifications (97\%) and involved in part time general practice (92.5\%). Significant number $(21 \%)$ of GPs have not heard the term syndromic management of STDs. Almost three quarter (74\%) of GPs did not use any guideline for the management for STIs.

Only a minority of GPs has prescribed the recommended antibiotic combination for patients presenting with urethral discharge $(10 \%)$, vaginal discharge (16\%), non vesicular genital ulcers $(3.5 \%)$ and vesicular genital ulcers (35\%). Majority (55.5\%) of GPs have done condom promotion. Counselling on STI prevention was done only by about half (48\%) of the GPs.

Conclusions: Most GPs were not aware of the syndromic management of STDs. Diagnostic tests that have been carried out were not appropriate to the symptoms and recommended treatment was not prescribed for most of the syndromes. They were unfamiliar with the STI management guidelines.

Key words: STIs-Sexually Transmitted infections, GPs- General practitioners

Authors: corresponding author; ${ }^{1}$ Dr. W. C. J. K. Jayakody, MBBS, PgD Ven, MD; Consultant Venereologist, Teaching Hospital, Kurunegala, Email:wcjksovis@gmail.com

2 Prof.A. Pathmeswaran, MBBS, MSc, MD (Com.Med); Department of Public Health, Faculty of Medicine, University of Kelaniya, Ragama. ${ }^{3}$ Dr. C. D. Wickramasuriya, MBBS, Msc, MD (Com.Med) Dip.GUM (Lon); National STD/AIDS Control Programme, Colombo.

Acknowledgement: Members of college of general practitioners and independent medical practitioners, all the Consultant Venereologists of National HIV/AIDS Control Programme

Originality: This is an original work not published anywhere, presented at $21^{\text {st }}$ Annual Scientific Sessions of Sri Lanka College of Sexual Health and HIV Medicine 2016.

Conflict of interest: No conflict of interest

Submitted: 14.12.2016, Accepted: 28.12.2016

\section{Full article}

Introduction

Sexually transmitted infections are of major public health importance as they predominantly affect sexually active age groups who are the main work force of a country. Complications of infection, stigma and discrimination attached to them, facilitation of the transmission and acquisition of HIV infection are major sequelae of STIs, if not detected and properly treated early ${ }^{1}$. Emergence of high levels of antibiotic 
resistance to some STIs has also been reported owing to inappropriate use of antibiotics $^{2}$. The assessment of practices and knowledge of GPs in contributing to the control and spread of STIs by early diagnosis and recommended treatment is very important.

General practitioners are an important segment of primary care providers in Sri Lanka. They provide comprehensive medical care and preventive care to patients and their families. They provide continuity of care and act as an advisor to patient and their families in all health related matters ${ }^{3}$. They play a valuable role in the management of sexually transmitted infections (STIs) too.

In Sri Lanka, any doctor who has gained full registration with the Sri Lanka medical council is eligible to start a general practice. One does not need postgraduate qualification or specific training to start a general practice ${ }^{4}$. They engage in full time or part time practice. Patients are free to select the doctor or the hospital as they wish. There is no strict referral procedure from primary care to secondary and tertiary care ${ }^{5}$.

It is estimated that $60-85 \%$ of patients with STIs seek treatment in the private sector ${ }^{6,7}$. Although a significant proportion of patients with STIs are seen and treated by GPs, so far, no studies had been done on the GPs knowledge or their practices related to the care given for STIs in Sri Lanka. Studies done in other countries have shown that GPs lack the correct knowledge on STIs.

As it is observed that a significant proportion of patients with STIs attend private sector, the services provided by GPs in the overall care should be assessed in order to provide a quality assured service. Most of the time, GPs manage patients with STIs clinically without going for the investigations due to money availability and time constraints. Syndromic management is a suitable option under this circumstance. Therefore, the assessment of correct knowledge on syndromic approach of STDs is beneficial for improvement of care rendered by them.

This study was carried out to describe the knowledge and practices related to the management of patients presenting with symptoms and signs of sexually transmitted infections (STD Syndromes) among general practitioners in the District of Gampaha and to identify the gaps.

\section{Method}

The study was carried out in Gampaha district in Western province. Gampaha is identified as a district rich with both public and private sector health care institutions. Study population was the general practitioners who have had MBBS, RMP qualifications or any other foreign qualification and registered at the SLMC and engaged in providing medical care for a fee. Two hundred GPs were assessed through pretested, self-administered questionnaire in English language. Sample size was calculated by using $95 \%$ confidence level and $5 \%$ confidence limit.

As there was no database or register for GPs for Gampaha district, a sampling frame was prepared using several available resources. There were 493 GPs in the list. GP centers were plotted on a geographical map of Gampaha district. The regions where there were more than seven GP centers were identified from the map. For logistical reasons, data collection was restricted to these areas. Total of 200 GPs who were willing to participate in the study were visited and given the questionnaires.

Epi data software was used for data entry. Data were processed and analyzed using SPSS 16. Ethical clearance was obtained from the ethical clearance committee, Faculty of Medicine, University of Kelaniya, Sri Lanka.

\section{Results}

Majority of the study population was male (76\%) and part time practitioners (92.5\%) with less than 10 years of experience in general practice (62\%). (Table 1). Fifty four percent of the study population was in the age range of $36-45 y e a r s$ with a median age of 42 years. It is significant that one fifth of GPs were seeing over 40 patients per day. Almost all the GPs were MBBS degree holders (97\%). 
Table1: Socio-demographic profile of GPs ( $N=200)$

\begin{tabular}{lrr}
\hline variable & Category & Freq (\%) \\
\hline Age in years & $26-35$ & $26(13.0)$ \\
\cline { 2 - 3 } & $36-45$ & $108(54.0)$ \\
\cline { 2 - 3 } & $46-55$ & $52(26.0)$ \\
\cline { 2 - 3 } & $56-65$ & $9(4.5)$ \\
\cline { 2 - 3 } & $66-75$ & $3(1.5)$ \\
\hline Sex & $>76$ & $2(1.0)$ \\
\cline { 2 - 3 } & Male & $152(76.0)$ \\
\hline Professional & Female & $48(24.0)$ \\
\cline { 2 - 3 } qualifications & MBSS PG & $63(31.5)$ \\
\cline { 2 - 3 } & MBBS & $131(65.5)$ \\
\cline { 2 - 3 } & RMP & $1(0.5)$ \\
\hline Duration of & Other & $5(2.5)$ \\
\cline { 2 - 3 } practice in & $<10$ & $124(62.0)$ \\
\cline { 2 - 3 } years & $11-20$ & $56(28.0)$ \\
\cline { 2 - 3 } & $21-30$ & $13(06.5)$ \\
\cline { 2 - 3 } & $>31$ & $6(03.0)$ \\
\cline { 2 - 3 } & Not & $1(0.5)$ \\
\hline
\end{tabular}

Significant number (21\%) of GPs have not heard the terminology of syndromic management of STDs. Out of the GPs who had heard about syndromic management, the knowledge on correct etiology for each syndrome was poor. Almost three quarter (74\%) of GPs did not use any guideline for the management for STIs and 15\% did not know that there is a guideline for STI management in Sri Lanka.

Fig 1: Management of STD patients by GPs

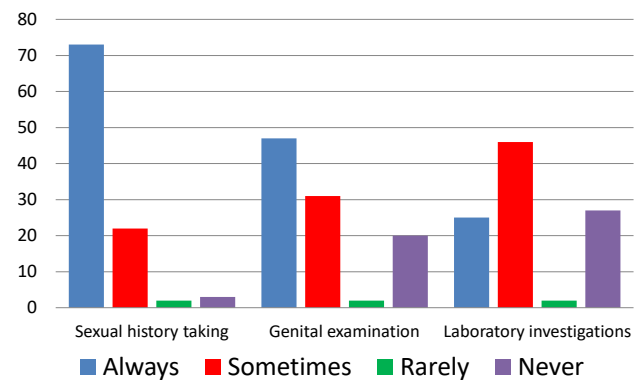

Although a significant number of GPs (73.5\%) were always keen to take sexual history, less than half of the GPs had always done genital examination (47\%). Laboratory investigations were done by only $72 \%$ of GPs. (Fig. 1) Of them, $52 \%$ and $42 \%$ have not done relevant laboratory investigations for urethral discharge and vaginal discharge, respectively. Most of the time, they have carried out UFR, urine culture, VDRL, FBC and FBS for patients with STI symptoms. Only a minority of GPs has prescribed the recommended antibiotic combination for patients presenting with urethral discharge $(10 \%)$, vaginal discharge $(16 \%)$, non vesicular genital ulcers (3.5\%) and vesicular genital ulcers (35\%).

Condom promotion was mentioned by $80 \%$ of GPs while $55.5 \%$ of GPs said they always promoted condom. Less than half of GPs (47.5\%) always counseled on STI prevention and $22 \%$ of them never did it. Sixty five percent said they refer patients to government STD clinics for further care.

\section{Discussion}

Syndromic management represents a simple, feasible treatment strategy for resource-poor settings. The immediate treatment avoids further transmission and complications that can occur as a result of loss to follow-up. Additionally, it minimizes the cost of laboratory testing and potentially avoids false negatives or positives that might come from poor laboratory settings and differences in sensitivity and specificity ${ }^{1}$. Because of these advantages, syndromic approach is a very useful method to adopt in the private sector where there are no laboratory facilities at hand.

In the present study, a significant proportion had insufficient knowledge on syndromic management or the terminology of syndromic management of STDs. After three decades of the introduction of syndromic approach by WHO, quite a few Sri Lankan GPs knew about it. Similar findings have been obtained in Pakistan where $82 \%$ of private and public sector health care providers were not aware of the terminology of "syndromic management of STDs" ${ }^{\prime 8}$.

Their knowledge on common etiology of main syndromes was also poor. Main etiology of urethral discharge and genital ulcer syndromes according to syndromic guideline 
were mentioned by $17 \%$ and $8.5 \%$ of GPs respectively .The study clearly showed the knowledge gap with respect to etiology of syndromes. This finding is not limited to Sri Lanka alone. Health care providers in other resource poor settings were also not aware on the correct etiology of the STI syndromes ${ }^{9,10}$ .Only $44 \%$ of Pakistan doctors have mentioned the correct etiology for urethral discharge and cervical discharge ${ }^{9}$.

Though guidelines are developed, management of STIs are not done accordingly by most of the GPs. Mere availability of a guideline does not reflect effective management of STIs because clinicians may not adhere to the standard guidelines due to lack of training or unawareness. In the present study almost three quarter of GPs (74\%) did not use any guideline to manage STIs and 15\% of GPs did not know about it. Even though the local guideline was published about 10 years prior to the current study, only a few GPs (3.5\%) have used it. It is obvious that syndromic approach is not popular among current generation of GPs in Sri Lanka.

Sexual history taking is one of the key components in STI management. Majority of GPs (73.5\%) had always taken sexual history once a patient presented with STI symptoms in the present study. However detailed sexual risk assessment with respect to sexual preferences and practices were obtained by $52 \%$ and $48 \%$ of GPs respectively.

Anyhow, GPs' interests to examine the patients were less compared to history taking. Only less than half of the GPs always examined the patients complaining of STI symptoms. This may be due to lack of privacy in some GP settings to carry out proper genital examination or they were in rush with patient load. Almost one quarter of GPs (25\%) made for etiological diagnosis and most of the time others have not requested the investigations. It is obvious that they practiced a somewhat similar method to syndromic management even though they did not know the terminology. However their testing was not appropriate to identify the etiology. Unnecessary testing was very common. Their most favorite tests for STI diagnosis were UFR, Urine culture and VDRL. The similar finding was identified in the previous study done in UK. Majority of GPs (54\%) have done the urinalysis and mid stream urine for culture in patients with dysuria and urethral discharge in that study ${ }^{11}$. Fifty four percent of GPs in this study have also done UFR for patients presenting with urethral discharge. These results made the conclusion that GPs were not aware about the correct diagnosis test based on the etiology of STD syndromes. Majority of the GPs (81\%) have ordered tests from private labs and a significant number of GPs (43\%) have referred patients to government STD clinics for investigation and further management.

Recommended treatment according to National guideline for three main syndromes were mentioned by a few GPs. Over treatment, under treatment and incorrect treatment was common for all three syndromes. Almost equal number of GPs has used doxycycline (35\%) which is recommended for chlamydia and ciprofloxacin (33\%) which has already been abandoned due to resistance to gonorrhoea. The immediate treatment for men with urethral discharge was tetracycline, ciprofloxacin and amoxicillin in UK ${ }^{11}$. Majority (51.5\%) of GPs had prescribed metronidazole for patients with vaginal discharge. Forty four percent of GPs used acyclovir for management of vesicular genital ulcers however a few GPs (5\%) prescribed benzathine penicillin for the treatment for non vesicular genital ulcers. All these findings indicate that under treatment and inappropriate therapy is common among GPs. Addressing the preventive strategies during STI case management further enhance the comprehensive care. According to National syndromic guideline, STI prevention includes educate and counsel on STIs, promote and provide condoms and contact tracing and partner treatment. This study shows $89 \%$ of GPs have mentioned that they have discussed about partner treatment with the patient. Most of the GPs (41\%) asked the patient to inform the contact to attend clinic and over 
one third of GPs (35.6\%) asked patient to bring the contact. However their way of tracing partners was vague and there was no proper system to check whether the partner has turned up for the treatment. The same method has been followed by some Pakistan GPs too. Thirty three doctors (41\%) encouraged STD patients to inform their partners about risk to them and 28 (35\%) asked the patient to bring in their partners ${ }^{9}$. It is interesting that almost equal percentages have found in both studies. Even in government settings, the level of contact tracing is not at an acceptable level in Sri Lanka. There are many factors that may be contributing to this. Social and cultural factors play a major role in this.

Majority of GPs in the present study have promoted the condoms and counseled their patient on STI prevention. Approximately two third of GPs have always (62\%) referred to further care whereas half that number $(33 \%)$ has sometimes referred to government STD clinics. Approximately two third of GPs have referred their patients with STDs to government STD clinics. This result support the findings from the study done in NSACP that out of symptomatic STI patients, $44 \%$ had taken treatment from private doctors before coming to the STD clinics $^{12}$.Our GPs were more likely to refer for further care when compared to Indian study where only one fourth of HCPs $(26.03 \%)$ had referred their patient to specialists.

\section{Conclusion}

Most GPs were not aware of the syndromic management of STDs. Therefore they lacked in depth knowledge on syndromes and the steps of management in a setting where investigation facilities are not available. Diagnostic tests that have been carried out were not appropriate to the symptoms and recommended treatment was not prescribed for most of the syndromes. Most of them have tried to prevent further transmission and future acquisition of STIs for the index patient. They were unfamiliar with the STI management guidelines. Therefore, general practitioners should be educated on syndromic management to provide better STI services to the patient. User friendly information booklet on STIs should be distributed to GPs to follow during their consultations. Steps should be taken to update STI knowledge and guidelines for GPs in private sector.

\section{References}

1. World Health Organization. Integrating STI/RTI Care for Reproductive Health Sexually Transmitted and Other Reproductive Tract Infections: A Guide to Essential Practice. Geneva: WHO; 2005. Report No.: ISBN 9241592656.

2. Iyalomhe GBS, Iyalomhe SI, Eholor R. Antibiotic prescription and resistance: A contemporary literature review. International Journal of Medicine and Medical Sciences. 2011 December; 3(14): p. 376-380.

3. De Silva $N$. Lecture notes in family medicine. 2nd ed. Sri Lanka: Sarvodaya Vishva Lekha; 2006.

4. Ramanayake R P. Historical Evolution and Present Status of Family Medicine in Sri Lanka. J Family Medicine Primary Care, India. 2013 April-June; 2(2): p. 131-134.

5. Ramanayake R P, Perera D P, De Silva A H, Sumanasekara R D. Patient held medical record: solution to fragmented health care in Sri Lanka. The health. 2013 Apr; 3.

6. World Health Organization. Involving Private Medical Practitioners in TB and STD Control, Report of Informal Consultation. New Delhi: WHO; 2001.

7. Independent Medical Practitioners Association. Epidemiology of STI and History taking in STI. 1st ed. Colombo: IMPA; 2005.

8. Mohsin S, Magnus U, Shakila Z, Cecilia S. Knowledge, attitude and practices regarding HIV/AIDS and STDs among health care providers in Lahore,Pakistan. JAyub Med Coll Abbottabad. 2009 Apr; 21.

9. Khandwalla H, Luby S, Rahman S. Knowledge, attitudes, andpractices regarding sexually transmitted infections among generalpractitioners and medical specialists in Karachi, Pakistan. SexTransm Infect. 2000; 76: p. 383-385.

10. Stokes T, Bhaduri S, Schober P, Shukla R. GPs' management of genital Chlamydia: a survey of reported practice. Family Practice an International Journel. 1997 August; 14(6): p. 455-60.

11. Ainsworth J, Weaver T, Murphy S, Renton A. General practitioners' immediate management of men presenting with urethral symptoms. Genitourinary Medicine. 1996 September; 72: $p$. 427-430.

12. Mallikarachchi M K, Liyanage R, De Silva A H. Health care seeking behaviour among Central STD clinic attendees. In College of Venereologists; 2008; Colombo. 